\title{
Nanostructured all-Silicon Photodetector Pixels with Tailored Responsivity Spectra
}

\author{
Jasper J. Cadusch*a ${ }^{\text {, Jiajun Meng }}{ }^{\mathrm{a}}$ and Kenneth B. Crozier*a,b \\ ${ }^{\text {a }}$ Department of Electrical and Electronic Engineering, University of Melbourne, Victoria 3010, \\ Australia \\ ${ }^{\mathrm{b}}$ School of Physics, University of Melbourne, Victoria 3010, Australia \\ *caduschj@unimelb.edu.au, kenneth.crozier@unimelb.edu.au
}

\begin{abstract}
We experimentally demonstrate nanostructured silicon photodetectors which consist of subwavelength arrays of verticallyoriented waveguides etched into a P-I-N photodiode. Our device combines both spectral-filtering and photocurrentgeneration in one all-Si structure. We show that absorption and responsivity spectra of these nanophotonic devices can be tuned by appropriate geometric design.
\end{abstract}

Keywords: Nanophotonics, Photodetectors, Waveguide Arrays, High Contrast Gratings

\section{INTRODUCTION}

Conventional wavelength sensitive photonic devices such as color cameras or spectrometers combine broadband solidstate photodetectors with separate wavelength filtering components such as dye-based absorptive filters or diffraction gratings. Combining these functionalities into a single device is desirable for light-sensing applications where footprint, cost and weight are important factors, such as in handheld personal electronics or on lightweight unmanned aerial vehicles. Here we present a device where the spectral filtering and photon detection occur within the same patterned silicon nanostructure that consists of subwavelength vertical waveguide arrays (WGAs). We experimentally demonstrate the engineering of responsivity spectra of our silicon $P-I-N$ photodiodes via carefully tailoring the geometric parameters (i.e. waveguide width, $s$, and array period, $\Lambda$ ) of our WGAs. We also describe an analytic method that allows us to predict the wavelength of peak absorption for an infinite array of vertical slab waveguides. This method is adapted from previous work on high contrast gratings [1], where the transverse magnetic (TM) mode cut-off wavelengths, $\lambda_{\mathrm{c}}$, can be found by solving

$$
\frac{1}{n_{s i}} \tan \left(\frac{\pi s}{\lambda_{c}}\right)-\tan \left(\frac{\pi(\Lambda-s)}{\lambda_{c}}\right)=0
$$

TE mode cut-off can be found by replacing the first term's prefactor with $n_{S i}$, (complex refractive index of silicon).

\section{RESULTS AND DISCUSSION}

A cross-sectional schematic of the WGA pixel with equivalent lumped circuit is shown in Fig. 1a. The red, green and blue layers represent $p+, i(n-)$ and $n+$-doped silicon regions respectively. Light normally incident on the device can couple directly to waveguide modes without the need for a momentum-matching device. Finite element method calculations of the fractional absorption density, $\alpha$, for a WGA $(s=75 \mathrm{~nm}, \Lambda=300 \mathrm{~nm})$ illuminated with a normally incident, TM-polarized plane wave $(\lambda=430 \mathrm{~nm})$ are shown in Fig. $1 \mathrm{~b}$. Here the power absorption density is normalized by the total power of the incoming plane wave. From the absorption profile, symmetric guided modes can be seen to be excited in each waveguide. Whether these modes are supported by the WGA at a particular wavelength can be controlled by selection of $s$ and $\Lambda$ according to equation (1). Fig. 1c. shows an SEM of a fabricated WGA photodetector. Each waveguide has a width of 220 $\mathrm{nm}$. Standard fabrication processes (e beam- and photo-lithography, e-beam evaporation and reactive ion etching) are used, potentially allowing for low-cost fabrication via CMOS-type processes. Here eight WGAs with periods between $300 \mathrm{~nm}$ and $500 \mathrm{~nm}$ are fabricated. 

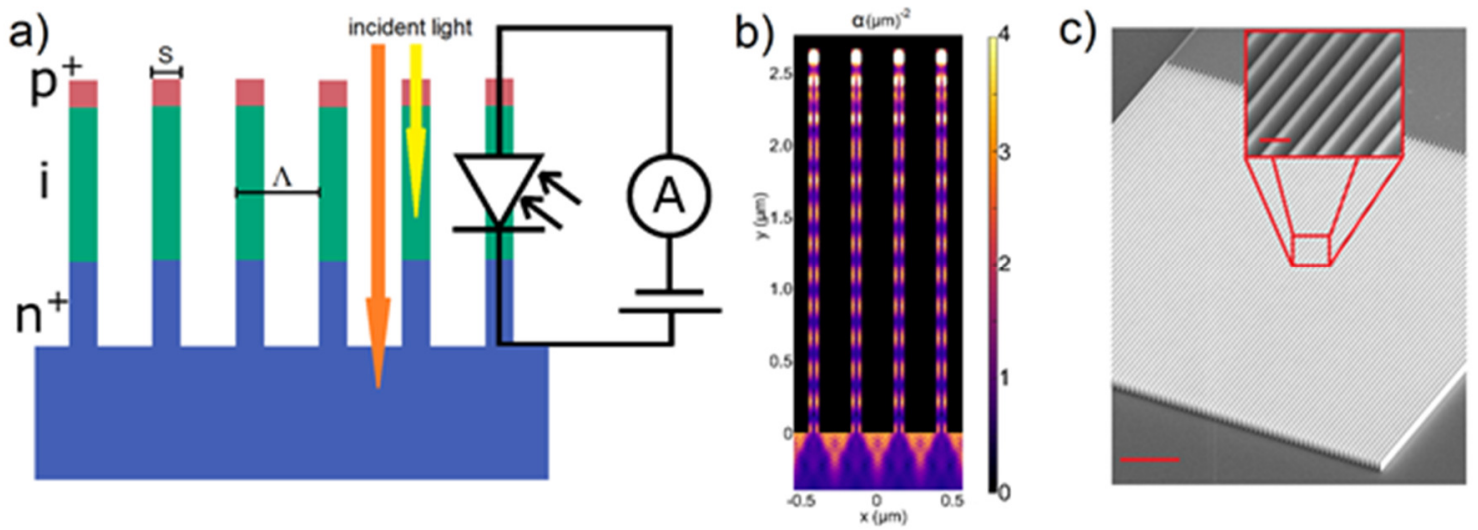

Figure 1a). Schematic of a WGA pixel. $p+, i(n-)$ and $n+$-doped regions are shown in red, green and blue respectively. b). Simulated fractional absorption density for a $300 \mathrm{~nm}$ period, $75 \mathrm{~nm}$ wide WGA. c). SEM of WGA pixel. Scale bar: $17 \mu$ m. Inset: zoom-in of WGA, scale bar: $0.5 \mu \mathrm{m}$.

We measure the responsivities of our fabricated WGA detectors by illuminating them with light of known power and wavelength. This is provided by a laser driven white light source coupled to a monochromator and is TE or TM polarized with respect to the WGA. The photocurrents from each pixel (for the two polarizations) vs wavelength at a reverse bias voltage of $1 \mathrm{~V}$ are recorded by a picoammeter (Keithley 6482). The normalized results (Fig. 2a\&b.) show an appreciable shift in peak responsivity wavelength with increasing array period. The HWHM of each detector is around $120 \mathrm{~nm}$. By using both TE and TM modes, good coverage of the visible range is achievable with just eight WGA pixels.
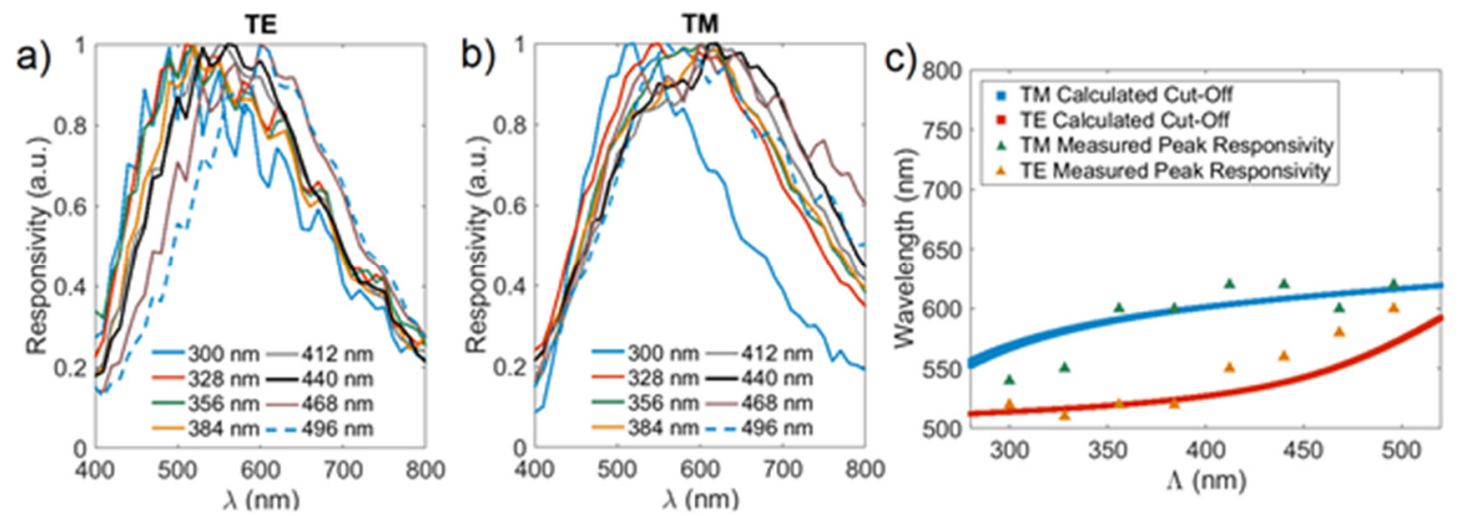

Figure 2. a\&b) Measured normalized responsivity spectra for TE a). and TM b). polarized light for a $220 \mathrm{~nm}$ wide WGA with varied periods. c) Cut-off wavelengths as a function of array period for even TM (blue) and TE (red) modes for a 220 nm wide WGA pixel.

The calculated cut-off wavelengths for TM2 and TE2 modes as a function of array period for a WGA consisting of 220 $\mathrm{nm}$ wide waveguides is shown in Fig 2c., superimposed with the measured peak responsivity wavelengths. There is good agreement between our simple modal model and each detector responsivity peak wavelength. The TE and TM modes are non-degenerate for this range of periods, hence coupling detectors with a polarizer can double the effective number of responsivities of a WGA pixel array. Furthermore, the cut-off wavelengths redshift with increasing array period.

In summary, we have demonstrated a compact CMOS-compatible nanophotonic device that functions as a visible spectrum photodetector. Each WGA pixel can be tuned to absorb different wavelengths, enabling engineering of the responsivity spectrum. Our approach could find use in portable consumer electronics for microspectrometry, colormatching, sample analysis or multi-spectral imaging.

\section{REFERENCES}




\section{University Library}

\section{- M I N E R VA}

\section{A gateway to Melbourne's research publications}

Minerva Access is the Institutional Repository of The University of Melbourne

Author/s:

Cadusch, JJ;Meng, J;Crozier, KB

Title:

Nanostructured all-Silicon Photodetector Pixels with Tailored Responsivity Spectra

Date:

2019-01-01

Citation:

Cadusch, J. J., Meng, J. \& Crozier, K. B. (2019). Nanostructured all-Silicon Photodetector Pixels with Tailored Responsivity Spectra. Mitchell, A (Ed.) RubinszteinDunlop, H (Ed.) AOS AUSTRALIAN CONFERENCE ON OPTICAL FIBRE TECHNOLOGY (ACOFT) AND AUSTRALIAN CONFERENCE ON OPTICS, LASERS, AND SPECTROSCOPY (ACOLS) 2019, 11200, SPIE-INT SOC OPTICAL ENGINEERING. https://doi.org/10.1117/12.2541213.

Persistent Link:

http://hdl.handle.net/11343/294879 